\title{
A Permeability Model for Coal and Other Fractured, Sorptive- Elastic Media
}

\section{Society of Petroleum Engineers Eastern Regional Meeting}

\author{
Eric P. Robertson \\ Richard L. Christiansen
}

\section{October 2006}

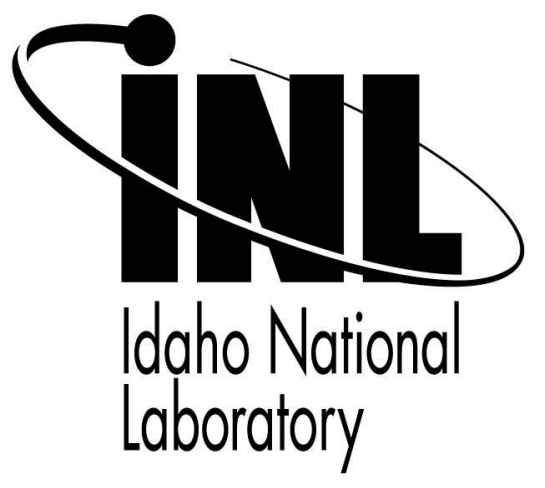

This is a preprint of a paper intended for publication in a journal or proceedings. Since changes may be made before publication, this preprint should not be cited or reproduced without permission of the author. This document was prepared as an account of work sponsored by an agency of the United States Government. Neither the United States Government nor any agency thereof, or any of their employees, makes any warranty, expressed or implied, or assumes any legal liability or responsibility for any third party's use, or the results of such use, of any information, apparatus, product or process disclosed in this report, or represents that its use by such third party would not infringe privately owned rights. The views expressed in this paper are not necessarily those of the United States Government or the sponsoring agency. 


\begin{abstract}
This paper describes the derivation of a new equation that can be used to model the permeability behavior of a fractured, sorptive-elastic media, such as coal, under variable stress conditions commonly used during measurement of permeability data in the laboratory. The model is derived for cubic geometry under biaxial or hydrostatic confining pressures. The model is also designed to handle changes in permeability caused by adsorption and desorption of gases from the matrix blocks. The model equations can be used to calculate permeability changes caused by the production of methane from coal as well as the injection of gases, such as carbon dioxide, for sequestration in coal. Sensitivity analysis of the model found that each of the input variables can have a significant impact on the outcome of the permeability forecast as a function of changing pore pressure; thus, accurate input data are essential. The permeability model can also be used as a tool to determine input parameters for field simulations by curve-fitting laboratory-generated permeability data. The new model is compared to two other widely used coal permeability models using a hypothetical coal with average properties.
\end{abstract}

\section{Introduction}

During gas production from a coal seam, as reservoir (pore) pressure is lowered, gas molecules, such as methane, are desorbed from the matrix and travel by diffusion to the cleat (natural fracture) system where they are conveyed to producing wells. Fluid movement in coal is controlled by slow diffusion within the coal matrix and described by Darcy flow within the fracture system, which is much faster than the contribution of diffusion. A coal formation is typically treated as a fractured reservoir with respect to fluid flow; meaning that the sole contributor to the overall permeability of the reservoir is the fracture system and the contribution of diffusion through the matrix to total flow is neglected. Coalbeds are unlike other non-reactive fractured reservoirs because of their ability to adsorb (or desorb) large amounts of gas, which causes swelling (or shrinkage) of the matrix blocks.

Coal has the capacity to adsorb large amounts of gases because of their typically large internal surface area, which can range from $30 \mathrm{~m}^{2} / \mathrm{g}$ to $300 \mathrm{~m}^{2} / \mathrm{g}$. ${ }^{1}$ Some gases, such as carbon dioxide, have a higher affinity for the coal surfaces than others, such as nitrogen. Knowledge of how the adsorption or desorption of gases affects coal permeability is important not only to operations involving the production of natural gas from coal beds, but also to the design and operation of projects to sequester greenhouse gases in coal beds. ${ }^{2}$ Laboratory measurements of permeability using coal samples can be used to gain insight into field-scale permeability changes and to determine key coal property values necessary for field-scale simulation.

A number of permeability models derived for sorptiveelastic media such as coals have been detailed in the literature and include those proposed by: Gray ${ }^{3}$ in 1987, Sawyer et al. ${ }^{4}$ in 1990, Seidle and Huitt ${ }^{5}$ in 1995, Palmer and Mansoori ${ }^{6}$ in 1998, Pekot and Reeves ${ }^{7}$ in 2003, and Shi and Durucan ${ }^{8}$ in 2003. These models were derived to mimic field conditions and assume a matrix-block geometry described as a bundle of vertical matchsticks under a uniaxial stress regime. ${ }^{6,9}$

However, in the laboratory, permeability is typically measured using hydrostatic (biaxial) core holders, which apply a single confining pressure to all external points of the core inside the holder. This is obviously different than the stress conditions encountered in the field, which are typically characterized as being under uniaxial stress. Moreover, on a bench-scale, coal matrix blocks may be better approximated by cubic instead of matchstick geometry as will be discussed later in this paper. A recent study ${ }^{10}$ compared the accuracy of three field-permeability models when applied to laboratorygenerated, sorption-affected permeability data and found that none of the three was able to accurately match the data. A model specifically derived for laboratory coreflooding conditions would be expected to provide a more reasonable match of permeability results.

This paper describes the derivation of a new model that describes the permeability behavior of a fractured, sorptiveelastic media, such as coal, under typical laboratory conditions where common radial and axial pressures are applied to a core sample during permeability measurements. The new model can be applied to fractured rock formations where the matrix blocks do not contribute to the porosity nor to the permeability of the overall system, but where adsorption and desorption of gases by the matrix blocks cause measurable swelling and shrinkage and thus affect permeability.

\section{Cleat Geometry}

Fig. 1 is a photograph of the ends of two different coal samples used by Robertson ${ }^{11}$ in his permeability experiments. Note the clear cleat markings on both samples. The geometry of the matrix blocks does not appear to cleanly fit a regular description; nevertheless, the cleats are generally oriented orthogonally for each of the coal samples in the photograph. Because of the apparent orthogonality of the cleat system, the selection of a cubic geometry for the matrix blocks appears to be a reasonable choice.

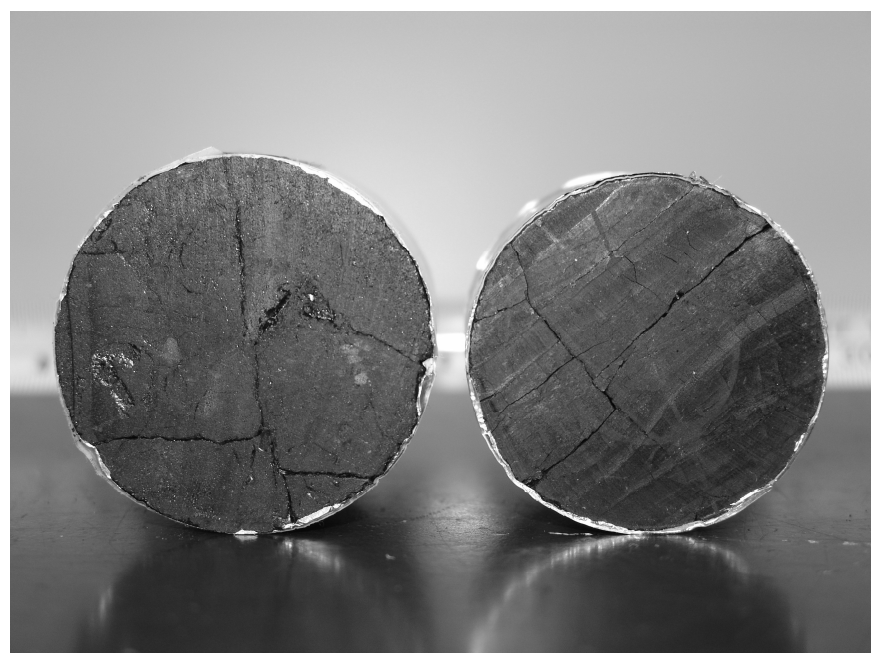

Fig. 1 - The image on the left is of Gilson coal and the image on 
the right is of Anderson coal. Both samples have diameters of 2.0 inches $(5.08 \mathrm{~cm})$.

\section{Porosity Equation for Assumed Cubic Geometry}

The control volume for the assumed cubic nature of coal is outlined by the dashed lines in Fig. 2, with the dimension $a$ equal to the height, width, and depth of the coal matrix block and dimension $b$ is the width of the cleats separating the matrix blocks.

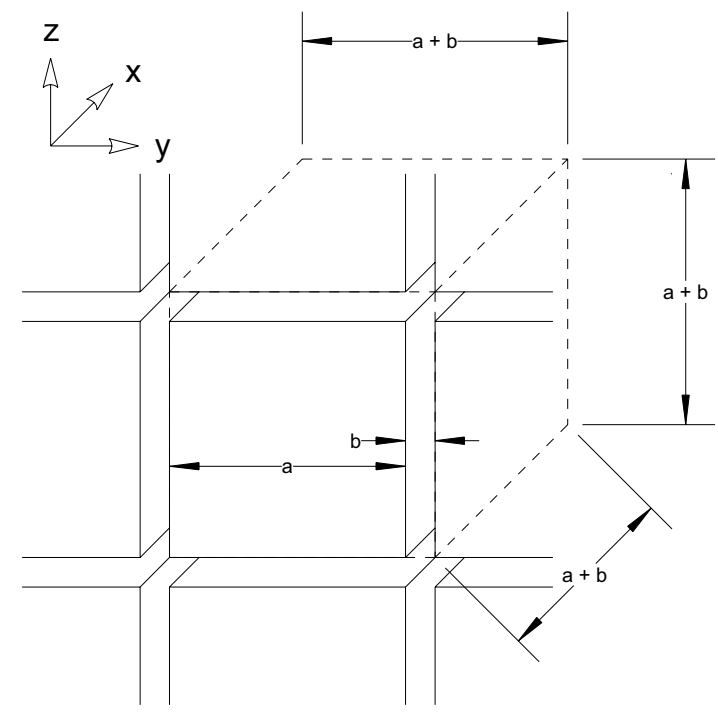

Fig. 2 - Schematic of idealized cleat system showing cubic matrix blocks (not to scale).

The following expression is derived in Appendix A for porosity (Eq. A-3) based on the cubic geometry shown in Fig. 2:

$\frac{a}{b}=\frac{3}{\phi}$

This equation compares favorably with results published by Reiss $^{12}$ and will be useful in the development of a permeability equation as will be shown hereafter.

\section{Permeability Equation for Fluid Flow through a Fractured Medium}

The following expression for permeability based on a cubic geometry and hydrostatic confining pressure is derived in Appendix B (Eq. B-10). It is a function of fracture width $b$ and fracture spacing $a$, and based on the work by Janna ${ }^{13}$ and Carmen: ${ }^{14}$

$k=\frac{b^{3}}{12 a}$

Permeability can be calculated using the above equation if the cleat spacing and fracture width were known. Permeability can also be measured directly through field tests or laboratory experiments, but being able to forecast changes in permeability is a greater need than calculating a one-time value. An equation that relates changes in permeability with respect to changes in pressure that has easily or commonly measured input parameters is the goal of this paper.

Permeability is a much stronger function of the cleat width $b$ than the matrix block width $a$ because of the cubic exponent attached to $b$. A simplifying assumption is to consider only the changes in $b$ due to changes in pressure. Taking the derivative of Eq. 2 with respect to pressure and letting $b$ be a function of pressure results in the following equation:

$\frac{d k}{d p}=\frac{3 b^{2}}{12 a} \frac{d b}{d p}$.

The term $d b / d p$ in the above equation represents the change in fracture width due to an infinitesimal change in pressure. The following sections discuss the factors that affect cleat width as a function of changes in pressure.

\section{Factors that Affect Cleat Width}

The goal of this section is to derive an expression for $\Delta b / \Delta p$ that can replace the $d b / d p$ term in Eq. 3. Although fracture width is a basic coal parameter, it is difficult to measure with any degree of accuracy even in laboratory specimens. In order to predict changes in permeability, the physics involved with changes in cleat width need to be mathematically described.

There are three different factors that contribute to changes in cleat width as different stresses are applied to a substrate such as coal: 1) pore volume compressibility $\left.\Delta b_{f}, 2\right)$ matrix compressibility governed by mechanical elastic moduli $\Delta b_{m}$, and 3) sorption-induced strain $\Delta b_{s}$. Each of these additive factors will be discussed in the following subsections and equations that can be used to relate changes in cleat width with changes in pressure due to each of these factors will be presented.

Pore Volume Compressibility. In the case being considered, the pore volume is equal to the volume of the fracture system; and pore volume compressibility is equal to the fracture compressibility, $c_{f}$. The compressibility of the fracture system is dependent on changes in both confining pressure and pore pressure.

Walsh ${ }^{15}$ gave the following general relationship between net stress $\sigma$, total stress $\sigma_{t}$, and pore pressure $p_{p}$ :

$\sigma=\sigma_{t}-s \cdot p_{p}$

where $s$ is a constant for elastic materials (such as coal). McKee et al. ${ }^{16}$ used the above relationship in their work involving stress-dependent permeability in coals, but set $s$ equal to unity with the caveat that this assumption might not be rigorously true. McKee et al. also state that the total stress $\sigma_{t}$ is a constant caused by the overburden. With these assumptions made, net stress can be defined as the difference between the overburden pressure $p_{o b}$ and the pore pressure $p_{p}$ :

$\sigma=p_{o b}-p_{p}$

The change in net stress is equal to the difference between the change in overburden pressure and the change in pore pressure:

$$
\Delta \sigma=\left(p_{o b}-p_{o b_{0}}\right)-\left(p_{p}-p_{p_{0}}\right) .
$$

Fracture compressibility is defined as the change in porosity with a change in stress: $:^{17,18}$

$c_{f} \equiv \frac{-1}{\phi_{0}} \frac{\Delta \phi}{\Delta \sigma}$.

Inserting Eq. 1 and Eq. 6 into Eq. 7 and rearranging to solve for the change in cleat width results in the following 
equation that relates the change in fracture width with respect to a change in pressure due to fracture compressibility:

$\Delta b_{f}=-b_{0} c_{f}\left\lfloor\left(p_{o b}-p_{o b_{0}}\right)-\left(p_{p}-p_{p_{0}}\right)\right\rfloor$

Mechanical Elastic Moduli. Depending on the material under consideration, changes in pore pressure can have a significant impact on fracture width due to the compressibility of the matrix blocks. The bulk modulus is the inverse of the elastic compressibility when an object is surrounded by fluid and a uniform stress is applied in all directions (hydrostatic pressure) and is defined as the negative change in volume associated with an increase in hydrostatic pressure ${ }^{19}$ or conversely as the positive change in volume associated with a decrease in hydrostatic pressure:

$$
K \equiv \frac{\Delta p_{\text {hydrostatic }}}{-\Delta V / V_{0}} \text {. }
$$

where $\Delta V$ is the change in volume of the object and $V_{0}$ is the initial volume of the object. In the case of coal matrix blocks, each block is, in reality, predominantly surrounded by pore space and held in place by a limited number of contact points with adjacent blocks and the initial concept described in Fig. 2 still holds. Because of this, changes in pore pressure would appear to have a much greater impact on the elastic compressibility of the matrix blocks than changes in confining pressure. To simplify resulting equations, we assume that changes in fracture width due to the mechanical elastic moduli of the matrix blocks is solely a function of changes in pore pressure and is independent of changes in overburden or confining pressure. Rearranging the preceding equation and making the pressure substitution results in

$$
-\frac{V-V_{0}}{V_{0}}=\frac{p_{p}-p_{p_{0}}}{K}
$$

In addition, volumetric mechanical strain $\varepsilon_{v m}$ is defined as the change in volume with respect to the original volume. ${ }^{19}$ The above equation can then be rewritten as

$$
-\varepsilon_{v m}=-\frac{V-V_{0}}{V_{0}}=\frac{p_{p}-p_{p_{0}}}{K} \text {. }
$$

The bulk modulus $K$, can be rewritten in terms of Young's modulus $E$ and Poisson's ratio $v^{19}$

$$
K=\frac{E}{3(1-2 v)} \text {. }
$$

Combining the two previous equations yields

$$
-\varepsilon_{v m}=\frac{3(1-2 v)\left(p_{p}-p_{p_{0}}\right)}{E} \text {. }
$$

It has also been shown that for isotropic media, the volumetric strain can be closely approximated by three times the linear strain. ${ }^{20}$ On a large scale, coal is not isotropic because of its layered characteristic, but on a small (matrix block) scale, there is evidence that coal behaves in an isotropic manner and that linear strain in all directions is equivalent to onethird of the volumetric strain, ${ }^{21}$ which supports the assumption that coal is an isotropic medium on a small scale. Substituting linear strain $\left(\varepsilon_{l m}\right)$ for one-third the volumetric strain results in the following equation: $-\varepsilon_{l m}=\frac{(1-2 v)\left(p_{p}-p_{p_{0}}\right)}{E}$.

The linear mechanical strain $\varepsilon_{l m}$ is defined as the change in length $\Delta a$ divided by the original length $a_{0}$ :

$\varepsilon_{l m} \equiv \frac{\Delta a}{a_{0}}=\frac{a-a_{0}}{a_{0}}$.

A positive change in the length of a matrix block results in an equal, but negative change in the cleat width; such that $\Delta a=-\Delta b$. Making these substitutions into Eq. (14) and rearranging to solve for the change in cleat width $\Delta b$ results in the following equation that relates changes in fracture width with respect to a change in pressure due to elastic mechanical properties of the coal:

$\Delta b_{m}=\frac{a_{0}(1-2 v)\left(p_{p}-p_{p_{0}}\right)}{E}$.

Sorption-Induced Strain. Gases are adsorbed onto the coal surfaces with increasing pore pressure and desorbed from the coal surfaces with decreasing pore pressure. The adsorption or desorption of gas molecules causes the length of the matrix block $a$ to either increase or decrease respectively. Sorption-induced strain, therefore, is solely a function of pore pressure and is not dependent on changes in overburden pressure. Sorption is used to mean either adsorption or desorption of gases from the surfaces of the coal matrix. Actual coal strain measurements due to the sorption of gases have not been readily available in the past, but recent advancements allow a more rapid measurement of sorption-induced $\operatorname{strain}^{22,23}$ making its measurement much easier. A change in the length of a matrix block results in an equal but opposite change in the cleat width; such that $\Delta a_{s}=-\Delta b_{s}$.

The change in the length of a matrix block $\Delta a_{s}$ is defined by the shape of the sorption-induced strain curve. A Langmuir-type equation with the following form has been shown to satisfactorily model sorption-induced strain curves in coal: ${ }^{22}$

$\varepsilon_{l s}=\frac{S_{\max } p_{p}}{p_{L}+p_{p}}$

where the Langmuir strain $S_{\max }$ is a constant representing the strain at infinite pore pressure and the Langmuir pressure $p_{L}$ is another constant representing the pore pressure at which the measured strain is equal to one-half $S_{\max }$.

The linear strain caused by sorption of gases, $\varepsilon_{l s}$, can be defined in terms of the difference between two points on the sorption-induced strain curve modeled using the above equation:

$$
\begin{aligned}
& \varepsilon_{l s}=\frac{S_{\max } p_{p}}{p_{L}+p_{p}}-\frac{S_{\max } p_{p_{0}}}{p_{L}+p_{p_{0}}} \Rightarrow \\
& \varepsilon_{l s}=\frac{S_{\max } p_{L}}{\left(p_{L}+p_{p_{0}}\right)\left(p_{L}+p_{p}\right)}\left(p_{p}-p_{p_{0}}\right) .
\end{aligned}
$$

As before,

$\varepsilon_{l s} \equiv \frac{\Delta a_{s}}{a_{0}} \Rightarrow \Delta a_{s}=a_{0} \cdot \varepsilon_{l s}$

By combining the previous two equations we obtain an expression for the change in $a_{s}$ as a function of pore pressure: 
$\Delta a_{s}=\frac{a_{0} S_{\max } p_{L} \Delta p_{p}}{\left(p_{L}+p_{p_{0}}\right)\left(p_{L}+p_{p}\right)}\left(p_{p}-p_{p_{0}}\right)$.

But $\Delta b_{s}=-\Delta a_{s}$ and substituting this into the above equation results the following equation that relates changes in fracture width with respect to a change in pressure due to sorptiveelastic properties of the coal:

$\Delta b_{s}=\frac{-a_{0} S_{\max } p_{L}}{\left(p_{L}+p_{p_{0}}\right)\left(p_{L}+p_{p}\right)}\left(p_{p}-p_{p_{0}}\right)$.

Total Change in Fracture Width Due to Changes in Pressure Conditions. The total change in fracture width $\Delta b_{t}$ caused by changing pressure conditions is the sum of the change caused by fracture compressibility (Eq. 8), mechanical elasticity (Eq. 16), and sorption of gases (Eq. 21):

$$
\begin{aligned}
\Delta b_{t} & =b_{0} c_{f}\left(\Delta p_{p}-\Delta p_{o b}\right) \\
& +\frac{a_{0}(1-2 v)}{E} \Delta p_{p}-\frac{a_{0} S_{\max } p_{L}}{\left(p_{L}+p_{p_{0}}\right)\left(p_{L}+p_{p}\right)} \Delta p_{p} .
\end{aligned}
$$

\section{Equations to account for changes in permeability due to changes in pressure}

Substituting Eq. 22 into Eq. 3 for $\Delta b$ results in the following:

$$
\frac{\Delta k}{\Delta p}=\frac{3 b^{2}}{12 a} \frac{1}{\Delta p}\left\{\begin{array}{c}
b_{0} c_{f}\left(\Delta p_{p}-\Delta p_{o b}\right) \\
+\frac{a_{0}(1-2 v)}{E} \Delta p_{p} \\
-\frac{a_{0} S_{\max } p_{L}}{\left(p_{L}+p_{p_{0}}\right)\left(p_{L}+p_{p}\right)} \Delta p_{p}
\end{array}\right\} \text {. }
$$

Varying Overburden Pressure with Constant Pore Pressure. The special case of varying only the overburden pressure, while holding pore pressure constant, is useful for laboratory experiments because it can be used to interpret laboratory results. If pore pressure is held constant, Eq. 23 reduces to the following form:

$\frac{\Delta k}{\Delta p_{o b}}=\frac{3 b^{2}}{12 a}\left(-b c_{f}\right)=\frac{-3 b^{3}}{12 a} c_{f}$.

By substituting the equation for permeability (Eq. 2) in for the quantity $b^{3} /(12 \cdot a)$, the above equation becomes

$$
\frac{\Delta k}{\Delta p_{o b}}=-3 k c_{f} \quad \text { but as } \Delta p \rightarrow 0 \quad \frac{d k}{k}=-3 c_{f} d p_{o b} \text {. }
$$

Integrating the above equation results in

$$
\frac{k}{k_{0}}=e^{-3 c_{f}\left(p_{o b}-p_{o b_{0}}\right)} \text {, }
$$

which can be used to model laboratory permeability data when overburden pressure is varied and pore pressure is held constant.

Varying Pore Pressure with Constant Overburden. Laboratory results obtained under a varying pore pressure regimen can be applicable to actual field scenarios such as pressure depletion to produce methane from coal beds, enhanced coal bed methane production by the injection of nitrogen, and during carbon dioxide sequestration operations in coal beds. If overburden pressure is held constant, Eq. 23 reduces it to the following form:

$$
\frac{\Delta k}{\Delta p_{p}}=\frac{3 b_{0}{ }^{3}}{12 a} c_{f}+\frac{3 b_{0}{ }^{2}}{12}\left[\frac{1-2 v}{E}-\frac{S_{\max } p_{L}}{\left(p_{L}+p_{p_{0}}\right)\left(p_{L}+p_{p}\right)}\right] \ldots . .
$$

Multiplying the second term in the above equation by unity $(a / b \cdot b / a)$ results in:

$$
\frac{\Delta k}{\Delta p_{p}}=\frac{3 b_{0}{ }^{3}}{12 a} c_{f}+\frac{3 b_{0}{ }^{3}}{12 a} \frac{a}{b_{0}}\left(\frac{1-2 v}{E}-\frac{S_{\max } p_{L}}{\left(p_{L}+p_{p_{0}}\right)\left(p_{L}+p_{p}\right)}\right) \text {. }
$$

Recall from Eq. 1 that $a / b=3 / \phi$ and from Eq. 2 that $b^{3} /(12 \cdot a)=k$. Substituting these relationships into the above equation results in

$$
\frac{\Delta k}{\Delta p_{p}}=3 k\left[c_{f}+\frac{3}{\phi_{0}}\left(\frac{1-2 v}{E}-\frac{S_{\max } p_{L}}{\left(p_{L}+p_{p_{0}}\right)\left(p_{L}+p_{p}\right)}\right)\right] .
$$

Rearranging this equation to fit a form capable of integration and letting $\Delta p$ approach zero results in

$$
\frac{d k}{3 k}=c_{f} d p_{p}+\frac{3}{\phi_{0}} \frac{(1-2 v)}{E} d p_{p}-\frac{3}{\phi_{0}} \frac{S_{\max } p_{L}}{\left(p_{L}+p_{p_{0}}\right)} \frac{d p_{p}}{\left(p_{L}+p_{p}\right)} .
$$

The permeability equation can now be integrated and results in the following equation:

$$
\frac{k}{k_{0}}=e^{3\left\{C_{f}\left(p_{p}-p_{p_{0}}\right)+\frac{3}{\phi_{0}}\left[\frac{(1-2 v)}{E}\left(p_{p}-p_{p_{0}}\right)-\frac{S_{\max } p_{L}}{\left(p_{L}+p_{p_{0}}\right)} \ln \left(\frac{p_{L}+p_{p}}{\left.p_{L}+p_{p_{0}}\right)}\right)\right]\right\}} .
$$

\section{Incorporating Variable Fracture Compressibility.} Fracture compressibility is not necessarily constant but is often a function of net stress. ${ }^{10}$ McKee et al. ${ }^{16}$ offered the following expression for stress dependent, variable fracture compressibility:

$c_{f}=\frac{c_{0}}{\alpha\left(\sigma-\sigma_{0}\right)}\left[1-e^{-\alpha\left(\sigma-\sigma_{0}\right)}\right]$

where $c_{0}$ is the initial fracture compressibility, $\alpha$ is the fracture compressibility change rate, and $\sigma$ is the net stress applied to the system. Using the definition of net stress reasoned in the section on Pore-Volume-Compressibility, the above equation for stress dependent fracture compressibility can be re-written as

$$
c_{f}=c_{0} \frac{1-e^{-\alpha\left[\left(p_{o b}-p_{o b_{0}}\right)-\left(p_{p}-p_{p_{0}}\right)\right]}}{\alpha\left[\left(p_{o b}-p_{o b_{0}}\right)-\left(p_{p}-p_{p_{0}}\right)\right]} .
$$

Final Permeability Equations. Substituting the above equation for stress dependent fracture compressibility into the permeability equations for variable overburden pressure (constant pore pressure) and variable pore pressure (constant overburden), we arrive at the final permeability equations for use to model laboratory permeability changes in sorptive elastic media such as coal.

For variable overburden pressure (constant pore pressure)

$$
\frac{k}{k_{0}}=\exp \left[-3 c_{0} \frac{1-\exp \left(-\alpha \Delta p_{o b}\right)}{\alpha}\right] \text {........ }
$$

This equation can be useful in determining the value of the fracture compressibility constants $c_{0}$ and $\alpha$. This can be accomplished by measuring values of permeability while vary- 
ing only the overburden pressure and then choosing values for $c_{0}$ and $\alpha$ that best fit the measured data.

The fracture compressibility constants can then be included in the permeability equation for variable pore pressure and constant overburden pressure:

$\frac{k}{k_{0}}=e^{3\left\{c_{0} \frac{1-e^{\alpha\left(p_{p}-p_{p 0}\right)}}{-\alpha}+\frac{3}{\phi_{0}}\left[\frac{1-2 v}{E}\left(p_{p}-p_{p_{0}}\right)-\frac{S_{\max } p_{L}}{\left(p_{L}+p_{p_{0}}\right)} \ln \left(\frac{p_{L}+p_{p}}{p_{L}+p_{p_{0}}}\right)\right]\right\}}$.

\section{Discussion}

Understanding the dynamics of the physics involved with changes in permeability in sorptive-elastic media, such as coal, is of vital interest to those involved with optimizing production from coal bed natural gas fields, sequestering carbon dioxide in coal beds, or producing natural gas from some shale formations. Laboratory experiments can be designed to enlighten the engineer as to what processes contribute to project success and how they can best be manipulated to increase recovery or economic viability. The equations derived above are based on the conditions encountered in laboratory experiments designed to calculate permeability. An accurate understanding of how permeability can change during production and injection operations is very important.

Earlier permeability models proposed by others ${ }^{6,7,8}$ were designed for field conditions and not for the interpretation of laboratory-generated permeability data. ${ }^{10,11}$ This present model should help researchers to better understand the processes that influence permeability and to derive realistic values of important parameters such as fracture compressibility, initial porosity, and elastic mechanical moduli, that need to be included in field-wide reservoir simulations.

\section{Input Data and Model Sensitivity.}

Table 1 is a listing of all the input variables necessary for Eq. 34 and Eq. 35. Also included are possible values and ranges of each variable gleaned from the open literature.

Using the average values of these input parameters, a hypothetical permeability-pressure relationship can be plotted for nitrogen gas flowing through coal (see Fig. 3).

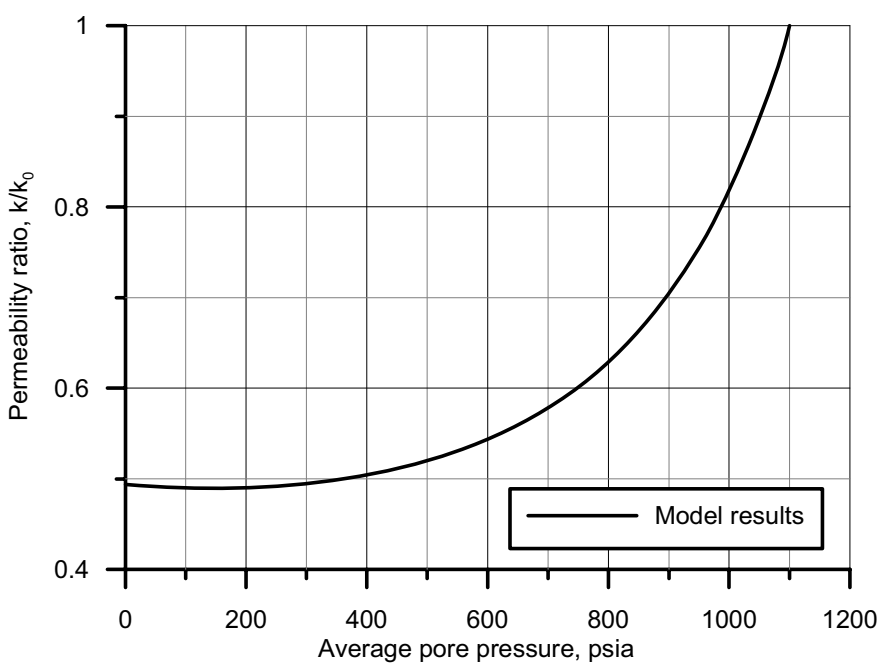

Fig. 3 - Model results using average input parameters from Table 1. For this case, nitrogen is the sorbing gas.

\section{Sensitivity of Model to Input Parameters}

Changes in some input parameters cause greater changes in model results than others. For example, porosity, sorption-

Table 1. Input variables required for permeability model and possible ranges of values.

\begin{tabular}{|c|c|c|c|}
\hline Coal property & Range & Reference & Average value \\
\hline Initial fracture compressibility, $c_{0}, \mathrm{psi}^{-1}$ & $\begin{array}{c}1.69 \mathrm{E}-4 \text { to } 1.88 \mathrm{E}-4 \\
18.0 \mathrm{E}-4\end{array}$ & $\begin{array}{l}\text { Robertson }{ }^{11} \\
\text { McKee et al. }{ }^{16}\end{array}$ & 7.19E-4 \\
\hline Compressibility change rate, $\alpha, \mathrm{psi}^{-1}$ & $\begin{array}{c}24.3 \mathrm{E}-4 \text { to } 37.5 \mathrm{E}-4 \\
29.5 \mathrm{E}-4\end{array}$ & $\begin{array}{l}\text { Robertson }{ }^{11} \\
\text { McKee et al. }\end{array}$ & $30.4 \mathrm{E}-4$ \\
\hline Initial porosity, $\phi_{0}$, fraction & $\begin{array}{c}0.001 \text { to } 0.005 \\
0.0005 \text { to } 0.0075 \\
0.03 \\
0.016 \\
0.024 \text { to } 0.028\end{array}$ & $\begin{array}{c}\text { Palmer and Mansoori } \\
\text { Young et al. }^{24} \\
\text { Sawyer et al. } \\
\text { Gash }^{25} \\
\text { Puri et al. }{ }^{26}\end{array}$ & 0.014 \\
\hline Poisson's ratio, $v$, fraction & $\begin{array}{c}0.23 \text { to } 0.40 \\
0.39 \\
0.32 \\
0.33 \\
0.20 \text { to } 0.50\end{array}$ & $\begin{array}{c}\text { Levine }^{21} \\
\text { Palmer and Mansoori }^{6} \\
\text { Gray }^{3} \\
\text { Seidle et al. }^{9} \\
\text { Shi and Durucan }\end{array}$ & 0.339 \\
\hline Young's modulus, $E$, psi & $\begin{array}{c}300,000 \text { to } 600,000 \\
124,000 \text { to } 445,000 \\
392,000 \\
500,000\end{array}$ & $\begin{array}{c}\text { Levine }^{21} \\
\text { Palmer and Mansoori }^{6} \\
\text { Gray }^{3} \\
\text { Seidle et al. }^{9}\end{array}$ & 393,500 \\
\hline Langmuir strain for nitrogen, $S_{\max }, \Delta L / L$, fraction & 0.00305 to 0.00196 & Robertson $^{11}$ & 0.00251 \\
\hline Langmuir pressure for nitrogen, $p_{L}$, psia & 1119.93 & Robertson $^{11}$ & 1119.93 \\
\hline
\end{tabular}


induced strain, and fracture compressibility are all important variables to correctly measure or estimate due to the large impact changes in these parameters have on model results; whereas Poisson's ratio and Young's modulus are somewhat less important.

The following sensitivity figures were plotted using data shown in Table 1. The "high" curves use the extreme upper values, and "low" curves use the extreme lower values, and the average curves use the average values.

Fracture Compressibility. Fig. 4 is a plot of the model using the low and high values for fracture compressibility (both $c_{0}$ and $\alpha$ ) as well as the model using the average values of all input parameters.

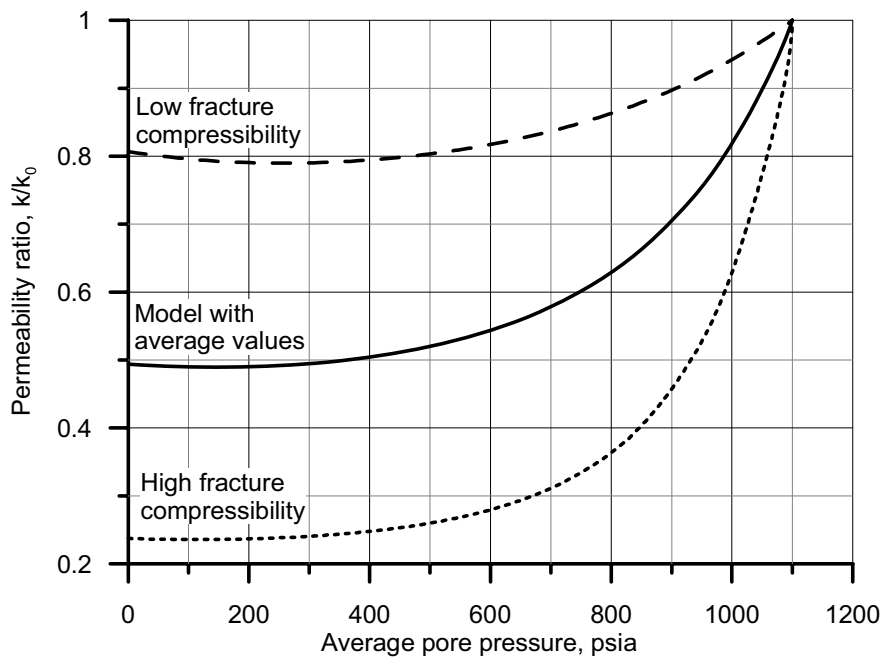

Fig. 4 - Sensitivity of the model to changes in fracture compressibility.

This figure shows that if the fracture compressibility is at the low end of the range given in Table 1, the drop in permeability as pore pressure decreases is significantly less than if the higher values were used. A good estimate for fracture compressibility is clearly needed before employing this model to match laboratory data or to forecast permeability reductions in the field. Fracture compressibility parameters can be accurately estimated by fitting Eq 35 to permeability data obtained while varying the confining pressure and holding pore pressure constant.

Porosity. Fig. 5 is a similar plot that shows the sensitivity of the model to different porosity values. Because porosity is in the denominator of the permeability equation, lower porosity values increase the relative impact of both the mechanicalelastic and sorptive-elastic properties of the coal. This is shown in the low porosity curve by the large decrease in permeability as pore pressure initially is lowered from 1100 psia, but as pore pressure continues to drop, the effect of nitrogen desorption becomes dominant and the permeability rebounds somewhat as pore pressure approaches zero.

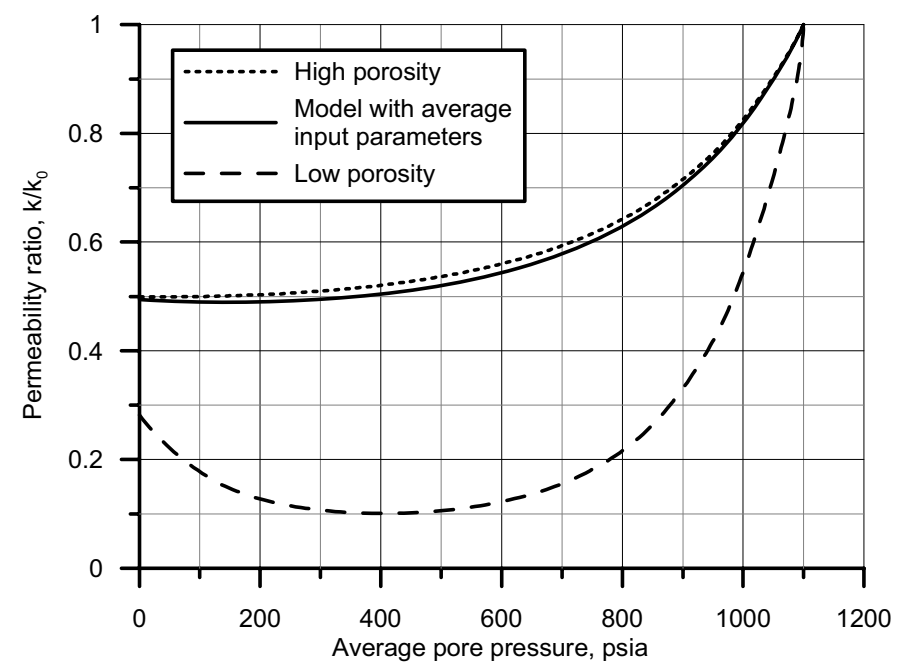

Fig. 5 - Sensitivity of the model to changes in porosity.

When a larger value for porosity is assumed, the model is dominated by the fracture compressibility term and the effect of mechanical-elastic and sorptive-elastic properties of the coal are minimized.

Mechanical-Elastic Properties. The sensitivity of the permeability model to different values of the mechanical-elastic properties of the coal (Poisson's ratio and Young's modulus) is shown in Fig. 6. Increases to Poisson's ratio and Young's modulus both result in higher permeabilities as pore pressure decreases.

Sorption-Induced Strain. Sorption-induced strain can have a dramatic impact on the permeability if the strain is large enough. Fig. 7 shows the sensitivity of the model to changes in sorption-induced strain data. Robertson and Christiansen ${ }^{23}$ have shown that there is a large difference between the strains induced by the adsorption of different gases in coal; they also showed that the amount of gas adsorbed is dependent on the coal rank. However, the variability of sorption-induced strain is much greater due to a change in gas than to a change in coal rank.
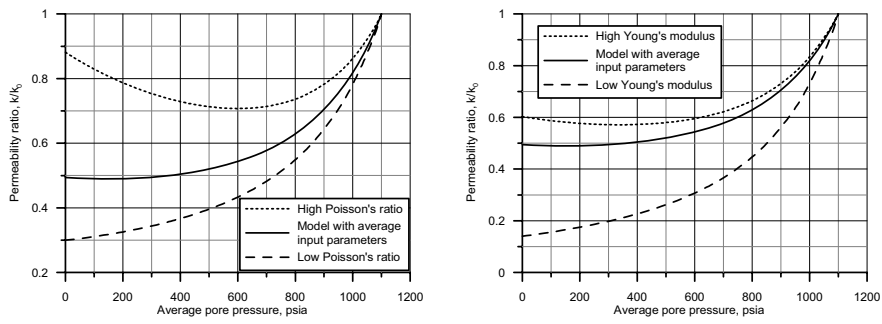

Fig. 6 - Sensitivity of the model to changes in the mechanical elastic properties of the coal: Poisson's ratio and Young's modulus. 


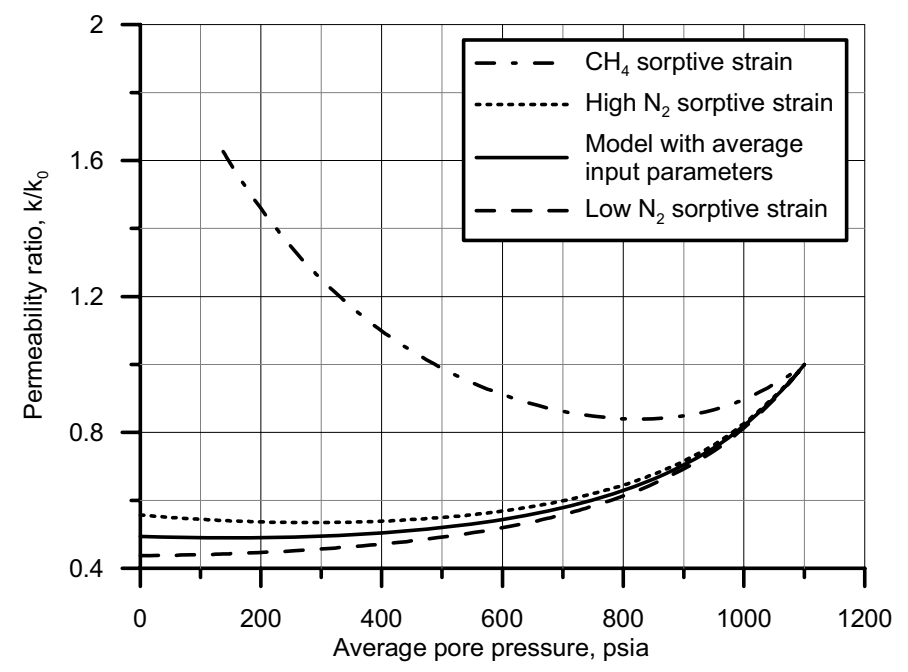

Fig. 7 - Sensitivity of the model to changes in the sorptive-elastic properties of the coal: $S_{\max }$ and $p_{L}$.

Fig. 7 not only shows the model sensitivity to changes of strain due to nitrogen desorption, but also compares permeability changes to a case where strain is induced by the desorption of methane where $S_{\max }=0.0087$ and $p_{L}=845 \mathrm{psi}^{11}$

Use as Tool to Predict Key Coal Properties. This model has been appropriately derived for conditions frequently used in laboratory-measured permeability test on coal samples. Key, hard-to-measure coal properties can be determined by fitting laboratory-measured permeability data with this model. For example, Young's modulus and Poisson's ratio are important parameters needed to accurately forecast permeability behavior during coalbed methane operations; ${ }^{6}$ yet, these values are difficult to measure and general values are typically used. If initial porosity, fracture compressibility, and sorption-induced strain were all fairly well known, the elastic moduli (Young's modulus and Poisson's ratio) could be determined by varying their values until the model reached a reasonable fit of the permeability data. These modeldetermined values could then be used in field-scale models to improve permeability forecasts.

Relative Contribution of Terms in Permeability Model. The permeability model as given in Eq. 35 contains three terms representing the contribution of fracture compressibility, mechanical elastic matrix strain, and sorptioninduced matrix strain to fracture width and permeability change. One might suspect that one could be more important to permeability change than the others or that one of the three could be a relatively small contributor to permeability change. Fig. 8 shows the relative contribution of each of the terms to the total permeability ratio calculated by the model.

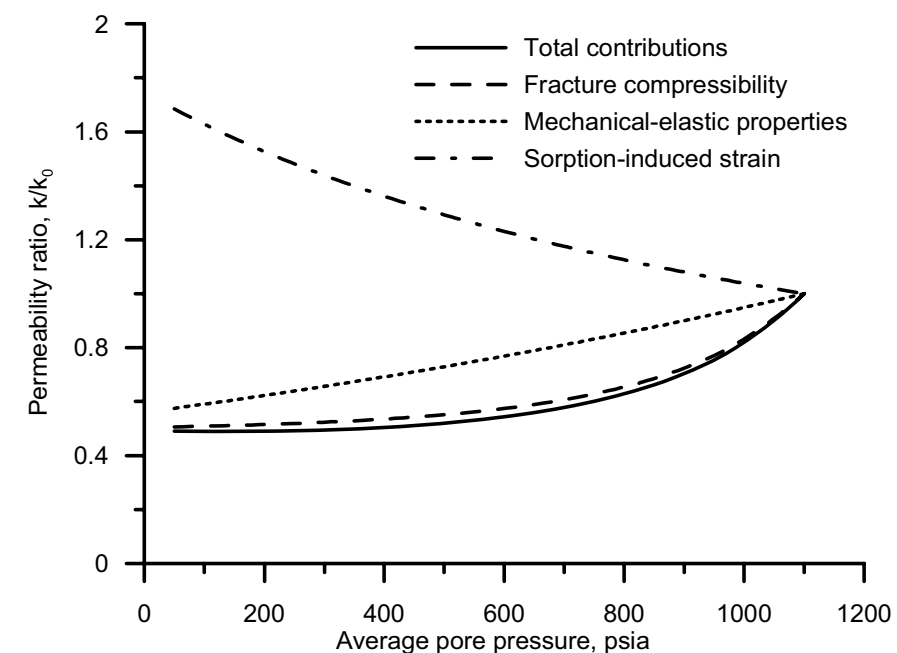

(a)

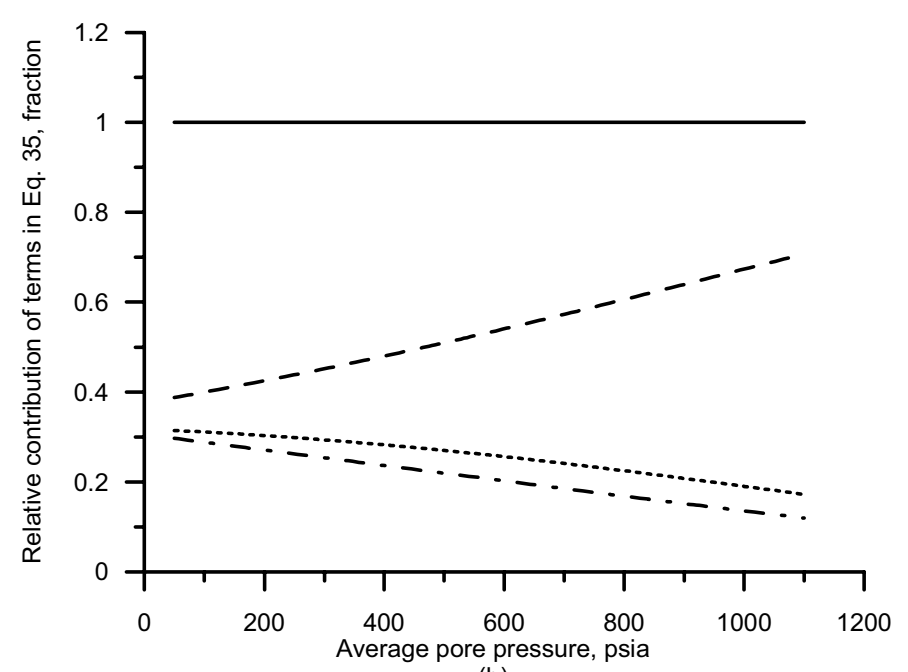

(b)

Fig. 8 - Plots showing the relative contributions of model terms to model output. For these figures, the model uses the average properties and the isotherm curve parameters for nitrogen found in Table 1.

For these particular conditions, the total permeability ratio curve in Fig. 8 (a) closely matches the permeability ratio resulting from solely the fracture compressibility term because the mechanical-elastic strain and the sorption-induced strain ten to counteract each other. Fig. 8 (b) shows that at higher pressures, the majority of the change in permeability results from the fracture compressibility term and as pressure is further reduced the contribution of each term is about the same.

The amount of strain contributed by the sorption of nitrogen compared to the other contributors is quite small, but is more notable when methane and carbon dioxide are sorbed. Fig. 9 compares the relative contributions of the model terms when methane is the sorbed gas and other parameters are the average parameters in Table 1. 


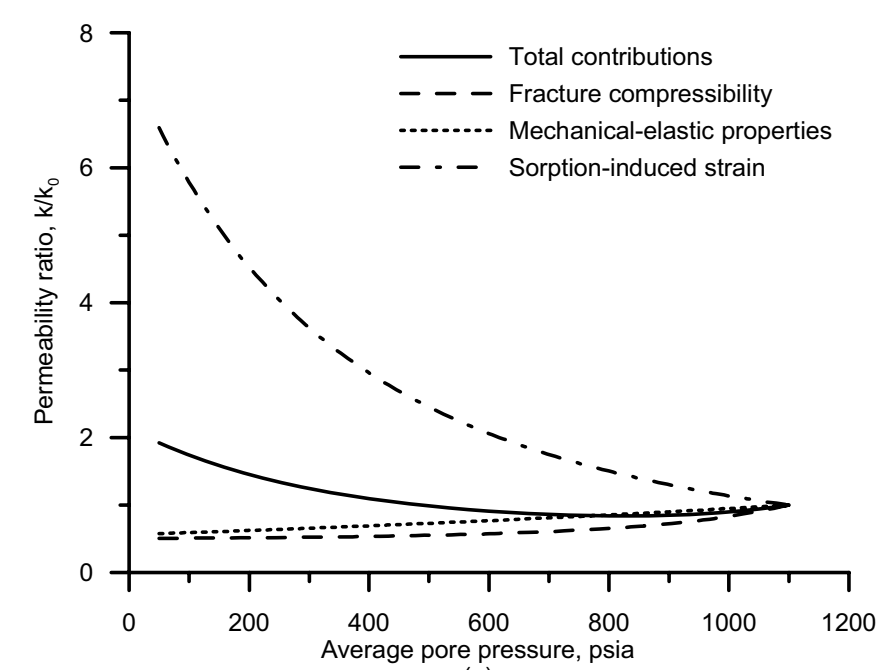

(a)

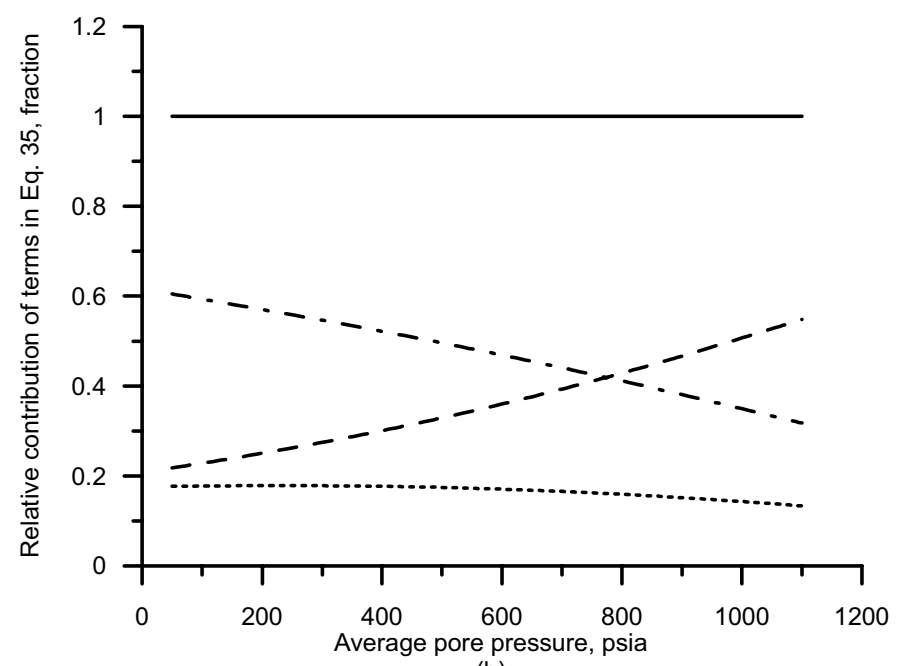

(b)

Fig. 9 - Plots showing the relative contributions of model terms to model output. For these figures, the model uses the average properties and the isotherm curve parameters for methane found in Table 1.

In Fig. 9 (b) the permeability model suggests that at high pore pressure, fracture compressibility is the dominant contributor to permeability change, but sorption-induced strain for methane becomes more dominant as pore pressure continues to decrease, which was not the case with the sorption of nitrogen (see Fig. 8).

Comparison of Model to Others. Other models for coal permeability changes have been proposed in the past. Prominent among those models are the Palmer-Mansoori model ${ }^{6}$ and the Shi-Durucan model. ${ }^{8}$

Both the Palmer-Mansoori model and the Shi-Durucan model were derived for matchstick-type fractured geometry under uniaxial strain conditions. Matchstick-geometry under uniaxial strain is equivalent to rectangular matrix columns packed together as a bundle of matchsticks with no horizontal fractures. The vertical (z-direction) overburden stress remains constant, while the horizontal ( $\mathrm{x}$ - and $\mathrm{y}$-directions) stresses vary with pore pressure. Both of these models were designed to match field stress conditions and may not be as applicable to laboratory permeability tests where the applied stresses are equal in all directions.

Compared to these two "field" models, the currently proposed model (Eq. 35) was derived to approximate laboratory coreflooding conditions and assumes a different stress regime (equal axial and radial stresses as opposed to uniaxial stress) as well as a different fracture geometry (cubic geometry as opposed to bundled matchsticks).

The Palmer-Mansoori permeability model can be expressed in the following form:

$\frac{k}{k_{0}}=\left[\begin{array}{c}1+\frac{(1+v)(1-2 v)}{E(1-v)} \frac{\left(p-p_{0}\right)}{\phi_{0}}+ \\ \frac{S_{\max }}{\phi_{0}}\left(\frac{(1+v)}{1-v}-3\right)\left(\frac{p}{p_{L}+p}-\frac{p_{0}}{p_{L}+p_{0}}\right)\end{array}\right]$

By modifying the Shi-Durucan permeability model to contain variable fracture compressibility, it can be expressed in the following form:

$\frac{k}{k_{0}}=e^{\left\{\frac{-3 C_{0}\left\{1-\exp \left[\alpha\left(p-p_{0}\right)\right]\right\}}{-\alpha\left(p-p_{0}\right)}\left[-\frac{v\left(p-p_{0}\right)}{1-v}+\frac{E \cdot S_{\max }}{(1-v)}\left(\frac{p}{p_{L}+p}-\frac{p_{0}}{p_{L}+p_{0}}\right)\right]\right\}}$

Eq. 35 is compared to both the Palmer-Mansoori and the Shi-Durucan models in Fig. 10. In this figure, nitrogen is the flowing fluid and the sorption-induced strain constants for nitrogen desorption are used.

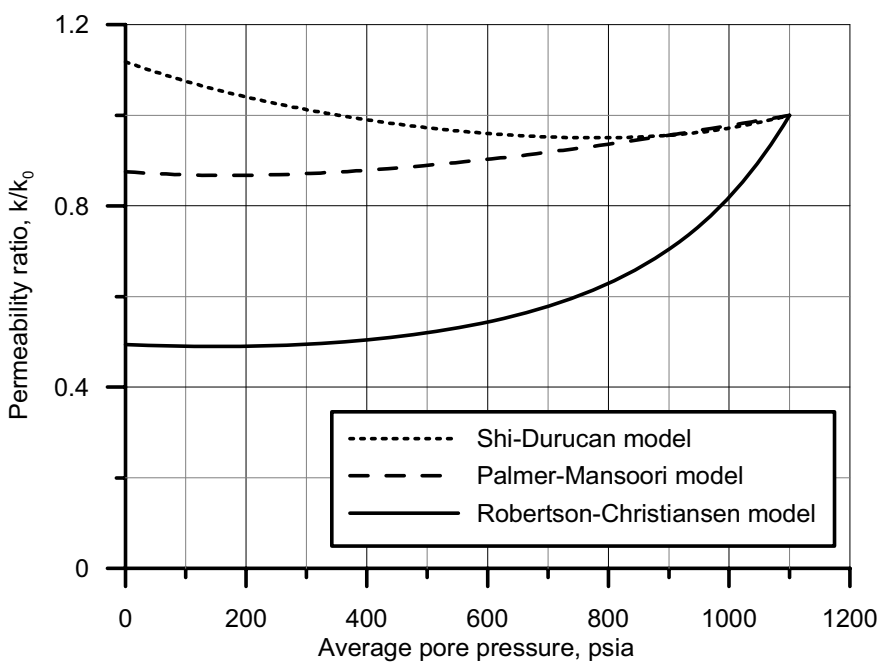

Fig. 10 - Model comparison of permeability changes for nitrogen flowing through an "average" coal core as pore pressure is lowered.

Altering the input to include the sorption-induced strain constants for methane desorption ${ }^{23}$ alters the relative shape of the models as seen in Fig. 11. 


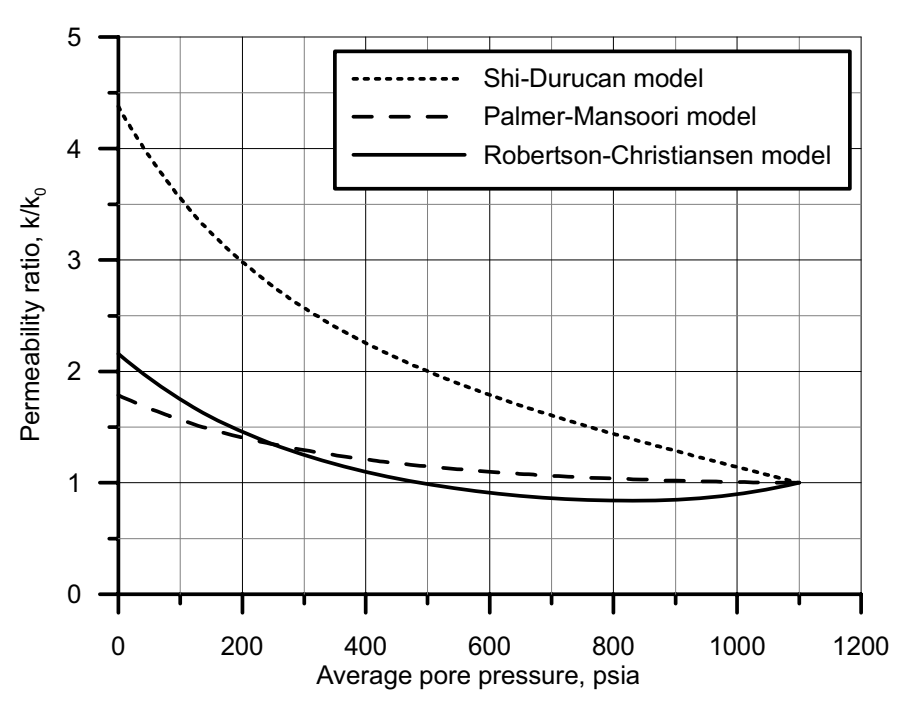

Fig. 11 - Model comparison of permeability changes for methane flowing through an "average" coal core as pore pressure is lowered.

Robertson and Christiansen ${ }^{10}$ analyzed and compared these two "field" models using laboratory generated coal permeability data and concluded that both models exaggerated the effect of sorption-induced strain on permeability in laboratory cores.

\section{Conclusions}

Conclusions of this work include the presentation of a new permeability model that can be used to model permeability changes in coal (and other substrates such as sorptive shale) as stresses change. In addition, the model can be used to model permeability changes caused by the injection of other gases such as carbon dioxide for sequestration in coal.

Sensitivity analysis of the model found that each of the input variables can have a significant impact on the outcome of the permeability forecast as a function of changing pore pressure. However, the permeability model can be used as a tool to determine some of the parameters by curve-fitting laboratory-generated permeability data. These model-determined values could then be used for field simulations with a greater degree of confidence.

The new model reduces the effect of sorption-induced strain on permeability compared to two "field" permeability models.

\section{Acknowledgements}

The authors would like to thank their respective companies and institutions for permission to publish this work. They also wish to thank Battelle Energy Alliance, LLC for the facilities and financial support to do this work.

\section{Nomenclature}

$a=$ width of matrix block or fracture spacing, $\mathrm{cm}$

$a_{0}=$ initial or original matrix block width, $\mathrm{cm}$

$\Delta a=$ change in matrix block width, $\mathrm{cm}$

$A=$ cross-sectional area of flow, $\mathrm{cm}^{2}$

$b=$ fracture width, $\mathrm{cm}$

$\Delta b=$ change in fracture width, $\mathrm{cm}$

$c=$ fracture compressibility, $\mathrm{psi}^{-1}$

$E=$ Young's modulus, psi $h=$ fracture height, $\mathrm{cm}$

$k=$ permeability, $\mathrm{md}$

$K=$ bulk modulus, psi

$L=$ length, $\mathrm{cm}$

$n=$ number of fractures in a given volume, dimensionless

$p=$ pressure, psia

$p_{L}=$ Langmuir pressure - pressure at which sorptioninduced strain equals $1 / 2$ the maximum, psia

$q=$ flow rate through single fracture, $\mathrm{mL} / \mathrm{s}$

$Q=$ total flow rate through a porous or fractured medium

$s=$ constant relating pressure to total stress; generally set equal to unity, dimensionless

$S_{\max }=$ linear strain at infinite pore pressure on an unconstrained sample, dimensionless

$v=$ velocity, $\mathrm{cm} / \mathrm{s}$

$V=$ volume, $\mathrm{mL}$

$\alpha=$ fracture compressibility change rate, dimensionless

$\varepsilon=$ strain, dimensionless

$\mu=$ viscosity, $\mathrm{cp}$

$v=$ Poisson's ratio, dimensionless

$\sigma=$ net stress, psi

$\tau=$ tortuosity

$\phi=$ porosity

\section{Subscripts}

$0=$ initial or original

$s=$ sorption-induced strain

$f=$ fracture

$l=$ linear

$m=$ mechanical elastic moduli

$o b=$ overburden

$p=$ pore

$t=$ total

$v=$ volumetric

\section{SI Metric Converstion Factors}
in. $\times 2.54^{*}$
$\mathrm{E}+00=\mathrm{cm}$
$\mathrm{psi} \times 6.894757 \mathrm{E}+00=\mathrm{kPa}$
* Conversion factor is exact.

\section{References}

1. Berkowitz, N.: Coal Science and Technology 7 - The Chemistry of Coal, Elsevier Science (1985) Table 3.16, p. 88.

2. "RECOPOL Workshop," Greenhouse Issues, Number 78 (June 2005) 5-7, www.ieagreen.org.uk, pp. 5-7.

3. Gray, I.: "Reservoir Engineering in Coal Seams: Part 1 - The Physical Process of Gas Storage and Movement in Coal Seams," paper SPE 12514, SPE Reservoir Engineering (February 1987) 28-34.

4. Sawyer, W.K. et al:: "Development and Application of a 3D Coalbed Simulator," paper CIM/SPE 90-119, presented at the 1990 International Technical Meeting hosted jointly by the Petroleum Society of CIM and the Society of Petroleum Engineers, Calgary, Alberta, Canada (10-13 June).

5. Seidle, J.P. and Huitt, L.G.: "Experimental Measurement of Coal Matrix Shrinkage Due to Gas Desorption and Implications for Cleat Permeability Increases," paper SPE 30010, presented at the 1995 International Meeting on Petroleum Engineering, Beijing, China (14-17 November 1995). 
6. Palmer, I. and Mansoori, J.: "How Permeability Depends on Stress and Pore Pressure in Coalbeds: A New Model," paper SPE 52607, SPE Reservoir Evaluation \& Engineering (December 1998) 539-544.

7. Pekot, L.J. and Reeves, S.R.: "Modeling the Effects of Matrix Shrinkage and Differential Swelling on Coalbed Methane Recovery and Carbon Sequestration, paper 0328, proc. 2003 International Coalbed Methane Symposium, University of Alabama, Tuscaloosa, Alabama (May 2003).

8. Shi, J.Q. and Durucan, S.: "Changes in Permeability of Coalbeds During Primary Recovery - Part 1: Model Formulation and Analysis," paper 0341 proc. 2003 International Coalbed Methane Symposium, University of Alabama, Tuscaloosa, Alabama (May 2003).

9. Seidle, J.P. et al.: "Application of Matchstick Geometry to Stress Dependent Permeability in Coals," paper SPE 24361, presented at the SPE Rocky Mountain Regional Meeting, Casper, Wyoming (18-21 May 1992).

10. Robertson, E.P. and Christiansen, R.L.: "Modeling Permeability in Coal Using Sorption-Induced Strain Data," SPE 97068, proc. 2005 SPE Annual Technical Conference and Exhibition, Dallas, Texas (10-13 October).

11. Robertson, E.P.: "Measurement and Modeling of SorptionInduced Strain and Permeability Changes in Coal," Ph.D. Dissertation, Colorado School of Mines, Golden, Colorado (2005).

12. Reiss, L.H.: The Reservoir Engineering Aspects of Fractured Formations, Gulf Publishing Company, Houston, Texas (1980) ISBN 0-87201-303-0.

13. Janna, W.S.: Introduction to Fluid Mechanics, Brooks/Cole Engineering Division, Monterey, California 93940, a division of Wadsworth, Inc. (1983) ISBN 0-534-01238-8, Eq. 5.20, p. 160.

14. Carmen, P.C.: "Fluid Flow Through Granular Beds," Trans., Inst. Chem. Eng., (1937) Vol. 15, 150-166.

15. Walsh, J.B.: "Effect of Pore Pressure and Confining Pressure on Fracture Permeability," Intl. J. Rock Mech. Min. Sci. Geomech. Abstracts (1981) 18, 5, 429-35.

16. McKee, C.R. et al.: "Stress-Dependent Permeability and Porosity of Coal and Other Geologic Formations," paper SPE 12858, SPE Formation Evaluation (March 1988) 81-91.

17. Amyx, J.W. et al.: Petroleum Reservoir Engineering - Physical Properties, McGraw-Hill, Inc. ISBN 07-001600-3 (1960) p. 291.

18. Slider, H.C.: Worldwide Practical Petroleum Reservoir Engineering Methods, PennWell Publishing Company, Tulsa, OK 74101, ISBN 0-87814-234-7 (1983) p. 117.

19. Petroleum Engineering Handbook, Third Printing, Howard B. Bradley Editor-in-Chief, Society of Petroleum Engineers, ISBN 1-55563-010-3 (1987) Chapter 51.

20. Robertson, E.P. and Christiansen, R.L.: "Measuring and Modeling Sorption-Induced Coal Strain," proc. 4th Annual Conference on Carbon Capture and Sequestration - DOE/NETL, paper 196, Alexandria, Virginia (2-5 May 2005).

21. Levine, J.R.: "Model Study of the Influence of Matrix Shrinkage on Absolute Permeability of Coal Bed Reservoirs," Coalbed Methane and Coal Geology, R. Gayer and I. Harris (eds), Geological Society Special Publication No. 109 (1996) 197-212.

22. Robertson, E.P. and Christiansen, R.L.: "Optically-Based Strain Measurement of Coal Swelling and Shrinkage," proc. 2004 International Coalbed Methane Symposium, paper 0417, University of Alabama, Tuscaloosa, Alabama (3-7 May).

23. Robertson, E.P. and Christiansen, R.L.: "Measurement of Sorption-Induced Strain," paper 0532, proc. 2005 International Coalbed Methane Symposium, University of Alabama, Tuscaloosa, Alabama (May 2005).
24. Young G. B. C. et al.: "An Analysis of Fruitland Coalbed Methane Production," paper SPE 22913, proc. 1991 SPE Annual Technical Conference and Exhibition, Dallas, Texas (6-9 October).

25. Gash, B.W.: "Measurement of "Rock Properties" in Coal for Coalbed Methane Production," paper SPE 22909, proc. 1991 SPE Annual Technical Conference and Exhibition, Dallas, Texas (6-9 October).

26. Puri, R. et al.: "Measurement of Coal Cleat Porosity and Relative Permeability," paper SPE 21491, presented at the SPE Gas Technology Symposium, Houston, Texas (23-25 January 1991). 


\section{Appendix A - Equation for Porosity for Cubic Matrix Blocks}

In this section, an equation for porosity is derived as a function of the cleat width and matrix block dimensions. The derivation results in a slightly different porosity equation than other permeability models (Palmer-Mansoori and ShiDurucan) because they assume matchstick-type geometry, while a cubic geometry is assumed here.

The effective porosity of the matrix block is assumed to be zero, leaving the fracture system to provide the only interconnected void space $\left(V_{f}\right)$. Porosity is then described by the following equation:

$$
\begin{aligned}
\phi & =\frac{V_{f}}{V_{t}}=\frac{V_{t}-a^{3}}{V_{t}}=\frac{(a+b)^{3}-a^{3}}{(a+b)^{3}} \\
& =\frac{3 a^{2} b+3 a b^{2}+b^{3}}{a^{3}+3 a^{2} b+3 a b^{2}+b^{3}} .
\end{aligned}
$$

Assuming that $b<<a$, we can eliminate all terms containing $b^{2}$ and $b^{3}$ resulting in

$\phi=\frac{3 a^{2} b}{a^{3}+3 a^{2} b}=\frac{3 b}{a+3 b}$.

Also, because $b<<a$, the denominator in the above equation reduces to $a$ and porosity becomes

$$
\phi=\frac{3 b}{a} \text {. }
$$

\section{Appendix B - Equation for Permeability of a Fractures System with Cubic Geometery}

In this section we derive an expression that relates permeability to the specific dimensions of the fractured system assumed in Fig. 2. Although a relationship between permeability and porosity has been shown by others, ${ }^{12}$ we believe that showing the derivation for this specific cubic-block geometry is of use to clearly understand the permeability-geometry relationship.

The fracture system shown in Fig. 2 is not drawn to scale and in a typical coal matrix/cleat system, the ratio of cleat width to cleat height is much lower than that depicted in the figure meaning that the contribution of the cleat intersections to total fluid flow can be neglected. Further, we assume that fluid flows in only the x-direction (into the page) in both the vertical and horizontal cleats. These simplifying assumptions allow the flow paths in Fig. 2 to be approximated by flow through rectangular ducts. Janna ${ }^{13}$ described the average velocity $(v)$ of laminar flow through a rectangular duct using the following equation:

$$
v=\frac{b^{2}}{12 \mu} \cdot \frac{\Delta p}{L}
$$

where $b$ is the width of the fracture, $\Delta p$ is the pressure at the inlet minus the pressure at the outlet, $L$ is the length of the duct, and $\mu$ is the viscosity of the fluid. The flow rate $(q)$ is equal to the velocity times the cross sectional area of flow: $q=v \cdot A$. But for a duct, the area, $A$, is equal to the height $(a)$ times the width $(b)$ or $A=a \cdot b$, resulting in the following equation for average velocity through a rectangular duct or fracture as a function of flow rate and fracture dimensions:

$$
v=\frac{q}{a b} \text {. }
$$
in $q=\frac{a b^{3} \Delta p}{12 \mu L}$

Now, let $n$ equal the number of fractures in a given volume of coal. The total flow, $Q$, through multiple fractures becomes $Q=\frac{n a b^{3} \Delta p}{12 \mu L}$.

Recall the following form of Darcy's Law for linear horizontal flow through a porous medium: ${ }^{17}$

$Q=\frac{k A \Delta p}{\mu L}$,

where $A$ is the area of flow representing multiple control volumes (see Fig. 2) and $k$ is the permeability of the porous or fractured medium.

The cross-sectional area of flow can be calculated from the matrix and fracture dimensions ( $a$ and $b$ ) and the number of fractures $(n)$.

$A=n(a+b)^{2}=n\left(a^{2}+2 a b+b^{2}\right)$

but the terms $2 a b+b^{2}$ can again be neglected because $b<<a$; and the preceding equation is reduced to

$A=n a^{2}$.

Substituting Eq. B-7 into Eq. B-5 gives Darcy's Law in terms of the dimensions and number of the matrix blocks as

$Q=\frac{k n a^{2} \Delta p}{\mu L}$.

Equating the flow through multiple fractures Eq. B-4 and the flow through a fractured medium Eq. B-8 gives

$\frac{n a b^{3} \Delta p}{12 \mu L}=\frac{k n a^{2} \Delta p}{\mu L}$.

Solving the preceding equation for permeability, $k$, yields an equation for permeability derived from flow through rectangular ducts and is applicable to a fractured medium of cubic geometry:

$k=\frac{b^{3}}{12 a}$.

Alternative Permeability Derivation Based on Carmen Equation. Carmen ${ }^{14}$ presented an equation for the permeability of a fractured media as a function of porosity $(\phi)$, tortuosity $(\tau)$, number of fractures per area $(n / A)$, and fracture height $(h)$ :

$k=\frac{\phi^{3}}{12 \tau\left(\frac{n}{A} h\right)^{2}}$.

Recognizing that porosity in our fractures system is the area occupied by the fractures divided by the total area, porosity can be written as

$\phi=\frac{n b h}{A}=\frac{n}{A} h b \Rightarrow \phi^{2}=\left(\frac{n}{A} h\right)^{2} b^{2}$.

Now, combining the two preceding equations results in

$k=\frac{\phi b^{2}}{12 \tau}$

Combining Eq. B-10 and Eq. B-13, yields an equation for permeability derived from the Carmen equation and based on the dimensions of the fractures and matrix blocks as well as tortuosity: 
$k=\frac{b^{3}}{4 \tau a}$.

Note that if $\tau=3$, then Eq. B-14 would be identical to Eq. B-10. 